\title{
Capacity Analysis for Secure Communication under CPA Attack in Smart Grid
}

\author{
Siqian $\mathrm{Liu}^{1}$, Bei Gou ${ }^{1}$, Hongxiang $\mathrm{Li}^{2}$ \\ 1 Department of Electrical and Computer Engineering \\ North Dakota State University \\ Fargo, ND 58108 \\ 2 Department of Electrical and Computer Engineering \\ University of Louisville \\ Louisville, Kentucky 40292
}

\begin{abstract}
In this paper, we extend the results of reliable and secure communication capacity requirements for eavesdropping attack to a more advanced chosen plain-text attack (CPA). Moreover, in order to calculate the power dynamic system's entropy, we analytically derived the formula for calculating the topological entropy of the system under the general differential algebraic equation (DAE) system model. Simulation results are presented to compare the capacity under eavesdropping and CPA based on DAE model and Alvarado model.
\end{abstract}

Keywords- Smart grid, CPA, DAE, capacity, topological entropy

\section{INTRODUCTION}

$\mathrm{R}$ ecent years, with the fast growing demand of energy resources, developing smart grid has become an urgent globe priority. Comparing to traditional power system, smart grid system has advantages of lower power consumption, high reliability and flexible network topology [1]. In a smart grid system, modern technologies in areas of power system, communication, control, and computer network are incorporated to monitor the operating status of the power system, optimize the power flow on branches, and reflect the time-varying power market price to consumers. In order to implement these functions, wide area network (WAN) [2] plays a center role to deliver the power network status to the operating center, transmit the smart meter readings, and update the instantaneous power market price for customers. Due to its importance, the security of WAN to defend against malicious attacks becomes a key issue to investigate. On the other hand, in order to meet the Quality of Service (QoS) requirements for users, we need to find the capacity of the communication in WAN.

In communication engineering, the capacity refers to the maximum information transmission rate with negligible probability of error and security is to ensure there is no information leakage during the transmission [3]. Traditionally, there are many studies in areas of communication and cryptography regarding the capacity requirement for security under different channels, for example: Additive White Gaussian Noise (AWGN) Channel, fading channel, multiple access channel and so on [4][5]. Most of these results are based on the assumptions that the information source is stationary, ergodic, and discrete-valued. In general, these assumptions are reasonable in traditional communication system such as broadcast and cellular network. However, smart grid system is highly dynamical due to all-time-varying power load thus the information source is no longer stationary and ergodic. Moreover, the system state information may be continuously valued. Therefore, the previous fundamental studies on secure communication will be no longer directly fitting to the smart grid system. As a matter of fact, there is still little research on reliable and secure communications in smart grid. In [6], the author established a simple information theoretic model for reliable and secure communication, several metrics in information theory have been re-defined to adapt to the discussion in dynamic system. But the discussion is only limited to the simplest eavesdropping attack. Moreover, the power system model used in [6] is Alvarado Model [7] that mainly focuses on the supply-demand relation and ignored to take the influence of the system's power load into account which is a key factor that determines system's dynamic. In order to accurately evaluate the influence to the communication capacity brought by of the system dynamics, differential algebraic model (DAE) [8][9] should be adopted. To the best of our knowledge, there has been no other related further research on the reliable and secure communication capacity in smart grid. For this reason, in this paper, we extend the result for reliable and secure communication capacity requirement under the chosen plain-text attack (CPA) [10][11] using a similar approach as in [6]. Moreover, we present our result for calculating the dynamic system's entropy under the general equation (DAE) model.

The paper is organized as the following: In Section II, the performance metric and the DAE model are defined. Then, we extend the security capacity from the eavesdropping attack to the CPA attack in section III. In section IV, we analytically derived the formula for calculating the system's entropy under a general form of the DAE model. Different simulation results under the Alvarado model and DAE model for both eavesdropping attack and CPA are presented in Section V. Finally, a conclusion is drawn in Section VI.

\section{SYSTEM MODEL}

We consider the power system dynamics as a discrete time system, in which the time is divided into time slots. In Figure 1, a general communication model in smart grid is shown. Without loss of generality, we assume Gaussian noises in the 
channel and the attacker in the middle only overhears the signal so it will not send any information through the channel. The state of the system $x(t)$ at any time slot $t$ is a function of the previous state and some random factors (load, number of users, electricity price, etc). In power system, the variation of the power load is the main reason of the system's dynamics. For the convenience of discussion, we assume the system state at time slot $t$ is only related to the state at time slot $t-1$. Thus, we can use the DAE to express the dynamics of the system as:

$$
\begin{gathered}
\boldsymbol{x} t+1=f \boldsymbol{x} t, \boldsymbol{w} t \\
\boldsymbol{x} t=g \boldsymbol{x} t, \boldsymbol{z} t \\
\boldsymbol{z} t=h \boldsymbol{x} t+\boldsymbol{e} t
\end{gathered}
$$

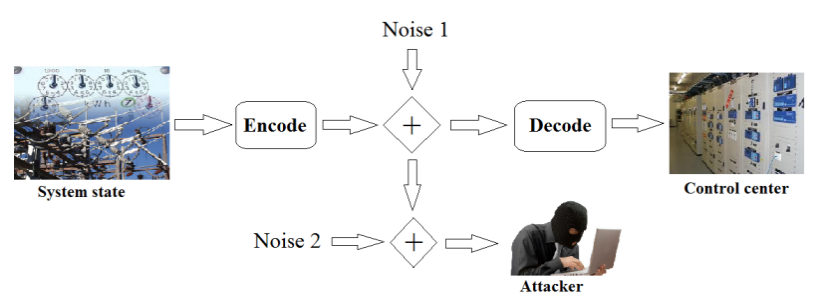

Figure 1: The Communication Model in Smart Grid

where $\boldsymbol{x} t$ is an $1 \times \mathrm{n}$ vector indicate the system state and $\mathbf{z} t$ is an $1 \times \mathrm{m}$ vector representing the measurement of the power system at time $t . \boldsymbol{w}(t)$ is the power load vector of the power system and $\boldsymbol{e}(t)$ is the measurement error at time $t . f$ is the function mapping the previous state vector and power load vector to the next state vector. In power system, we call $f$ as the power load forecasting. In practice, the system state vector $\boldsymbol{x} t$ cannot be measured directly (for example, the voltage phase at each bus, imaginary power flow on branches). Instead, by measuring some other parameter $\mathbf{z} t$, we can get the estimation of the state $\boldsymbol{x} t$.

Since the dynamic system presented in (1)-(3) may not be stationary and ergodic, the traditional measurement of the security using entropy is no longer suitable. Due to the distinctiveness of the dynamic system, we use the topological entropy $H X, f, g, h$ introduced in [12] as the measure which quantitatively represents the total amount of information for estimated message that asymptotically equals to the transmitted message in a time-varying system. Then we can define the security equivocation $\Delta$ as:

$$
\Delta=\frac{H X, f, g, h \mid Z}{H X, f, g, h}
$$

where $H X, f, g, h$ is the amount of information transmitted when there is no attacker in the system and $Z$ indicates the condition that the attacker exists. When $\Delta=1$, we say the communication in a dynamical system is reliable and secure. This is because $H X, f, g, h \mid Z=H X, f, g, h$ at this time, which means no information will be leaked when the attacker occurs. From the prospective of the information theory, we just need to find the region for $H X, f, g, h$ such that we can always find an encoding-decoding scheme such that the control center can reliably receive the system state information while the attacker can obtaining no information on system state. Formally, we have the following definition:

Definition: If the communication between transmitter and receiver assures the observability of the system state and satisfies $\Delta=1$, we say that the communication is reliable and secure.

\section{SECURE CAPACITY ANALYSIS}

We use $C_{R}$ and $C_{A}$ to denote the channel capacity from the transmitter to the destination and the attacker. According to the Proposition 1 in [6], the reliability and security of the communication under eavesdropping attack can be assured when $H X, f$ is smaller than the difference of the $\mathrm{C}_{\mathrm{R}}$ and $\mathrm{C}_{\mathrm{A}}$. However in the model of $C P A$, besides overhearing the channel, attacker can also query an oracle (or "magic box") with pre-selected plain-text and get the encrypted (encoded) message. Due to this ability, extra part of the transmitted information is needed to use to protect the message in CPA. The traditional deterministic public-key encryption is no longer safe. As a result, to ensure security under CPA, we need to use probabilistic encoding. Thus, in order to ensure the reliability and security under CPA attack, we have the following theorem:

Theorem 1: When the following inequality holds, then the reliability and security of the communication is ensured: in which

$$
H X, f, g, h \leq C_{R}-C_{A}-H_{\text {loss }}(X, f, g, h)
$$

$$
\begin{gathered}
C_{R}=B \log _{2}\left(1+\frac{P}{\sigma_{0}^{2}}\right) \\
C_{A}=B \log _{2}\left(1+\frac{P}{\sigma_{0}^{2}+\sigma_{1}^{2}}\right)
\end{gathered}
$$

where $\sigma_{0}^{2}$ is the variance of Gaussian noise between transmitter and receiver. $\sigma_{1}^{2}$ is the Gaussian noise between the transmitter and the attacker. $H_{\text {loss }}$ is the information loss brought by the probabilistic coding.

Proof: Since for the CPA, the attacker also does not send any information through the channel. As the case of eavesdropping, according to Proposition 1 in [6], we have:

$$
H X, f, g, h \leq C_{R}-C_{A}
$$

However, based on the definition of CPA, the attacker can build the table of possible messages and their corresponding cipher-texts. To find the decryption of some observed ciphertext, the attacker just need to look the cipher-text up in the table. As a result, public-key definitions of security under chosen-plaintext attack require probabilistic encryption [13]. Thus, comparing to eavesdropping, the only difference in capacity is brought by the probabilistic coding, which is $H_{\text {loss }}$. Thus, we have the theorem proved.m

Due to the different probability distribution chosen for coding scheme, the $H_{\text {loss }}$ has different forms. In particularly, if we choose the uniform distribution for the probabilistic coding, then we have the following corollary:

Corollary 1: When the probabilistic coding follows the uniform distribution, then the reliability and security of the communication is ensured:

$$
H X, f, g, h \leq C_{R}-C_{A}-\frac{1}{n} \log _{2} n
$$

where $n$ is the length of the coded message.

\section{SECURE CAPACITY UNDER DAE MODEL}

Even though we have obtained the reliable and secure capacity requirements for the communication in smart grid, however, it is still unknown how to calculate the topological entropy $H X, f$. With different power system model and 
assumptions, the expression for $H X, f$ will be varied. In [6], the author presents a formula for calculating the topological entropy under the linear Alvarado model. However, Alvarado model considers the power system dynamics mainly based on the view of supply-demand relationship and cannot accurately reflect the power load change in the network. In the following section, we derive the expression of $H X, f$ under a realization of the differential algebraic model.

In (3), we can re-write as:

$$
\boldsymbol{z} t=\begin{gathered}
z_{1} t \\
z_{2} t \\
\cdots \\
z_{m} t
\end{gathered}=\begin{gathered}
h_{1} \boldsymbol{x} t \\
h_{2} \boldsymbol{x} t \\
\cdots \\
h_{m} \boldsymbol{x} t
\end{gathered} \quad+\begin{aligned}
& e_{1} \\
& \cdots \\
& e_{m}
\end{aligned}=\boldsymbol{h} \boldsymbol{x} t+\boldsymbol{e}
$$

Without loss of generality, we can assume that measurement errors are independent and each $e_{i}$ follows the Gaussian distribution with zero mean and variance $\sigma_{e i}^{2}$. By using the weighted least square (WLS), method, the optimal estimation for $\boldsymbol{x} t$ is obtained by minimize the following cost function:

$$
J \boldsymbol{x} t=\left[\begin{array}{llll}
\mathbf{z} & t-\boldsymbol{h} \boldsymbol{x} t
\end{array}\right]^{T} R^{-1}\left[\begin{array}{llll}
\mathbf{z} & -\boldsymbol{h} \boldsymbol{x} t
\end{array}\right]
$$

where $R=E\left[\boldsymbol{e}^{T}\right]$ is the covariance matrix of the measurement errors. When $J(\boldsymbol{x} t)$ is minimized, its derivative becomes zero, so we have:

$$
\frac{\partial J \boldsymbol{x} t}{\partial \boldsymbol{x} t}=-H^{T} \boldsymbol{x} t \quad R^{-1} \boldsymbol{z} t-\boldsymbol{h} \boldsymbol{x} t=0
$$

where $H \boldsymbol{x} t=\frac{\partial \boldsymbol{h} x t}{\partial \boldsymbol{x} t}$ is the hessian matrix. In order to solve for (12), we use Newton's method and set the initial value as:

$$
\boldsymbol{x}^{0} t=\boldsymbol{x} t-1+g \boldsymbol{x} t-1, \boldsymbol{z} t-1
$$

so we have:

$$
\begin{aligned}
& \boldsymbol{x} t-\boldsymbol{x}^{0} t
\end{aligned}
$$

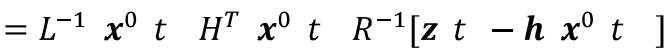

where $L \begin{array}{llllllll}\boldsymbol{x}^{0} & t & =H^{T} \boldsymbol{x}^{0} & t & R^{-1} H & \boldsymbol{x}^{0} t\end{array}$.

From (14), we can see that if the previous system estimation and the current measurements are known, then we can calculate the current system state estimates by (14). If we denote $F \boldsymbol{x}^{0} t=L^{-1} \boldsymbol{x}^{0} t \quad H^{T} \boldsymbol{x}^{0} t \quad R^{-1}$, then we can write (13) as:

$$
\boldsymbol{x} t=\boldsymbol{x}^{0} t+F \boldsymbol{x}^{0} t\left[\boldsymbol{z} t-\boldsymbol{h} \boldsymbol{x}^{0} t\right]
$$

Using Taylor expansion and neglect the high order terms, we get:

$$
\boldsymbol{x} t=I-\frac{\partial M}{\partial x^{0} t}+\frac{\partial F}{\partial x^{0} t} \mathbf{z} t \boldsymbol{x}^{0} t-M 0+F 0 \mathbf{z} t
$$

where $M \boldsymbol{x}^{0} t=F \boldsymbol{x}^{0} t \boldsymbol{h} \boldsymbol{x}^{0} t$. Similarly, we can write (13) as:

$$
\boldsymbol{x}^{0} t=I+\frac{\partial g}{\partial \boldsymbol{x} t-1} \boldsymbol{x} t-1+g 0, \mathbf{z} t-1
$$

For the second term in (17), since at the time $t-1$, the system state is zero, this means the system is not working thus the output for the measurement must be zero and $g 0,0=0$. In this way, equation (17) becomes:

$$
\boldsymbol{x}^{0} t=I+\frac{\partial g}{\partial x t-1} \boldsymbol{x} t-1
$$

Taking (18) into (16) and we have:

$$
\boldsymbol{x} t=I-\frac{\partial M}{\partial x^{0} t}+\frac{\partial F}{\partial x^{0} t} \boldsymbol{z} t \quad I+\frac{\partial g}{\partial x t-1} \boldsymbol{x} t-1-
$$

Using chain rules for $\frac{\partial M}{\partial x^{0} t}$ and $\frac{\partial F}{\partial x^{0} t}$ :

$$
M 0+F 0 \boldsymbol{z} t
$$

$$
\begin{aligned}
& \frac{\partial M}{\partial x^{0} t}=\frac{\partial M}{\partial x t-1} \frac{\partial x t-1}{\partial x^{0} t} \\
& \frac{\partial F}{\partial x^{0} t}=\frac{\partial F}{\partial x t-1} \frac{\partial x t-1}{\partial x^{0} t}
\end{aligned}
$$

Using (20) and (21) to substitute the corresponding terms in (19):

$$
\begin{aligned}
& \boldsymbol{x} t=I+\frac{\partial g}{\partial x t-1}-\frac{\partial M}{\partial x t-1}+\frac{\partial F}{\partial x t-1} I+\frac{\partial g}{\partial x t-1}^{-1} \mathbf{z} t \quad I+ \\
& \frac{\partial g}{\partial x t-1} \quad \boldsymbol{x} t-1-M 0+F 0 \boldsymbol{z} t
\end{aligned}
$$

In equation (16), since $\boldsymbol{z} t$ is the measurement vector which is deterministic. Let $A t-1=I+\frac{\partial g}{\partial x t-1}-\frac{\partial M}{\partial x t-1}+$ $\frac{\partial F}{\partial x t-1} I+\frac{\partial g}{\partial x t-1}^{-1} \boldsymbol{Z} t\left(I+\frac{\partial g}{\partial x t-1}\right) \quad$ and $\quad B t=$ $-M 0+F 0 \boldsymbol{Z} t$, we can write the equation (22) in the following form:

$$
\boldsymbol{x} t=A t-1 \boldsymbol{x} t-1+B t
$$

Based on (23), we have the following theorem for the topological entropy of the system:

Theorem 2: If $\lim _{t \rightarrow \infty} A t=A<\infty$ and ${ }_{0}^{\infty} B t d t<\infty$, then the topological entropy of the dynamic system $(1)(2)(10)$ can be represented as follow:

$$
H(X, f, g, h)={ }_{i=1}^{N} \log _{2}\left(\max \left(1, \lambda_{i}\right)\right)
$$

where $\lambda_{1}, \lambda_{2}, \ldots, \lambda_{N}$ are eigenvalues of the matrix $A$. Specifically, if the dynamic system tends to be stable, then the system has zero topological entropy.

Proof: Since $\lim _{t \rightarrow \infty} A t=A<\infty$ and ${ }_{0}^{\infty} B t d t<\infty$, for any given $\varepsilon>0$, whenever $t$ is large enough, we have $A t-$ $A<\varepsilon$. Thus, we can write the equation (23) as:

$\boldsymbol{x} t=A t-1-A \boldsymbol{x} t-1+A \boldsymbol{x} t-1+B t$

Since $\boldsymbol{x} t$ is composed by system parameters and each parameter in the system has a threshold, $\boldsymbol{x} t$ must be bounded. Assume $\boldsymbol{x} t \leq B$, then when $\mathrm{t}$ is large enough such that $A t-A<\varepsilon / B$, we have :

$$
\boldsymbol{x} t=\varepsilon_{0} / 2+A \boldsymbol{x} t-1+B t
$$

Also, as ${ }_{0}^{\infty} B t d t<\infty$, and $B t=-M 0+F 0 z t$ is measurable, we have:

$$
\lim _{t \rightarrow \infty} B t=0
$$

Equation (26) implies that if $t$ is large enough, then $B t<$ $\varepsilon_{0} / 2$. Thus, for any $\varepsilon_{0}>0$, there exists $t_{0}$ such that for $t>t_{0}$, equation (25) becomes:

$$
\boldsymbol{x} t=A \boldsymbol{x} t-1+\varepsilon_{0}
$$

According to Theorem 2.4.2 in [14], the topological entropy of the dynamic system in the form of (27) is:

$$
H X, f, g, h=H A=\log _{2}\left(\max \left(1, \lambda_{i}\right)\right)
$$

When the system tends to be stable, according to the definition of stability, we have $\lambda_{i}<1$ for any eigenvalue of $\mathrm{A}$. Thus:

so theorem 2 is proved.

$$
\left.H X, f, g, h={ }_{i=1}^{N} \log _{2}(1)\right)=0
$$

Even though equation (24) provides a general formula for calculating the topological entropy, however, during the 
computation, the forms of the hessian matrices $H \boldsymbol{x} t, \frac{\partial g}{\partial x t-1}, \frac{\partial M}{\partial x t-1}, \frac{\partial F}{\partial x t-1}$ are very complicated and time-consuming to calculate. For this reason, in practice, we always use a simplified DAE DC power system model:

$$
\begin{gathered}
\boldsymbol{x} t+1=F_{0} \boldsymbol{x} t+G_{0} \\
\boldsymbol{z} t=H_{0} \boldsymbol{x} t+e
\end{gathered}
$$

In this case, following the similar procedures as (11) to (23), we can get the system topological entropy for the DC power model when $G_{0}=0$ is:

$$
H X, f, g, h=H F_{0}={ }_{i=1}^{K} \log _{2}\left(\max \left(1, \lambda_{i}\right)\right)
$$
where $\lambda_{1}, \lambda_{2}, \ldots, \lambda_{N}$ are eigenvalues of the matrix $K$.When $G_{0} \neq 0, H X, f, g, h$ is infinite when $F_{0}$ is stable.

\section{Simulation Results}

. In our simulation, an IEEE 14-bus system infrastructure [16] is used as shown in Figure 2 with the total power 1. Based on it, the measurement matrix $H_{0}$ in equation (30) is obtained as shown in Figure 3. We provide the simulation results for the secure and reliable communication capacities under eavesdropping attack and CPA, respectively. For the $\mathrm{CPA}$, we assume the measurement and system state messages are encoded by the uniformly probabilistic coding. Moreover, in order to illustrate the difference in topological entropy due to the choice of system models, both Linear Alvarado Model and DAE Model are implemented in simulations.

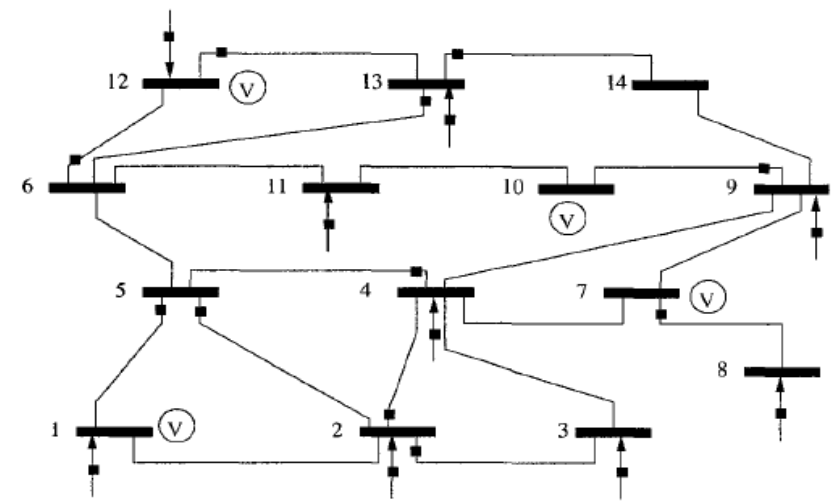

Figure 2: The IEEE 14-bus system [16]

$\mathrm{H}=$

$\begin{array}{rrrrrrrrrr}-1 & 0 & 0 & -1 & 0 & 0 & 0 & 0 & 0 & 0 \\ 4 & -1 & -1 & -1 & 0 & 0 & 0 & 0 & 0 & 0 \\ -1 & 2 & -1 & 0 & 0 & 0 & 0 & 0 & 0 & 0 \\ -1 & -1 & 5 & -1 & 0 & -1 & 0 & -1 & 0 & 0 \\ 0 & 0 & 0 & 0 & 0 & -1 & 1 & 0 & 0 & 0 \\ 0 & 0 & -1 & 0 & 0 & -1 & 0 & 4 & -1 & 0 \\ 0 & 0 & 0 & 0 & -1 & 0 & 0 & 0 & -1 & 2 \\ 0 & 0 & 0 & 0 & -1 & 0 & 0 & 0 & 0 & 0 \\ 0 & 0 & 0 & 0 & -1 & 0 & 0 & 0 & 0 & 0 \\ 1 & -1 & 0 & 0 & 0 & 0 & 0 & 0 & 0 & 0 \\ 0 & 0 & 0 & 0 & 0 & 1 & -1 & 0 & 0 & 0 \\ 0 & 0 & 0 & -1 & 0 & 0 & 0 & 0 & 0 & 0 \\ 1 & 0 & 0 & -1 & 0 & 0 & 0 & 0 & 0 & 0 \\ 1 & 0 & -1 & 0 & 0 & 0 & 0 & 0 & 0 & 0 \\ 0 & 0 & 1 & 0 & -1 & 0 & 0 & 0 & 0 & 0 \\ 0 & 0 & 0 & 0 & 1 & 0 & 0 & 0 & 0 & 0 \\ 0 & 0 & 0 & 0 & 1 & 0 & 0 & 0 & 0 & 0 \\ 0 & 0 & 0 & 0 & 0 & 0 & 0 & 1 & -1 & 0 \\ 0 & 0 & 0 & 0 & 0 & 0 & 0 & 0 & 0 & 0 \\ 0 & 0 & 0 & 0 & 0 & 0 & 0 & 0 & 0 & 0\end{array}$

Figure 3: The Measurement Matrix [16]

Under the Alvarado model, the power system state satisfies the following dynamics [15]:

$$
P_{g}=\left(T-b_{g}-c_{g} P_{g}-k E\right) / \tau_{g}
$$

$$
\begin{aligned}
P_{d}=\left(b_{d}\right. & \left.-c_{d} P_{d}-T\right) / \tau_{d} \\
E & =P_{g}-P_{d} \\
T & =-E / \tau_{T}
\end{aligned}
$$

where the $P_{g}$ and $P_{d}$ are the amount of power generated and consumed. $\mathrm{T}$ is the unit price of power and $\mathrm{k}$ is the power load (rate). $\tau_{g}, \tau_{d}, \tau_{T}$ are the rate controlling parameters of the supply, demand and the price. $b_{g}, c_{g}, b_{d}, c_{d}$, are the parameters for generator and consumers. The exact physical meaning can be checked in [15]. In the simulation, we assume the main influence of the power system is the power load. For this reason, we set $\tau_{g}=\tau_{d}=\tau_{T}=1, b_{g}=c_{g}=0.1, b_{d}=$ $c_{d}=0.05$. The secure capacity under eavesdropping and CPA are presented in Figure 4 and 5 (marked with "non- DAE") Comparing with the eavesdropping, we can see that the CPA attack has obvious lower capacity under the same power load, which is matched with the Theorem 1. Also, both results are implemented using the simplified DAE DC model in Figure 4 and Figure 5. The setting of the load forecasting matrices $F_{0}$ and $G_{0}$ can be found in [16]. Compare these results in Figures, we found that the topological entropy of the system under simplified DAE DC model are close to the results under Alvarado Model.

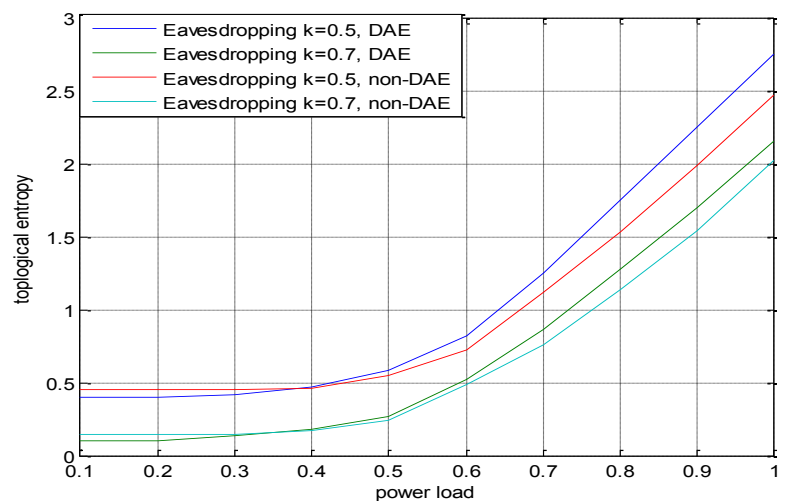

Figure 4: The eavesdropping secure capacity under linear models

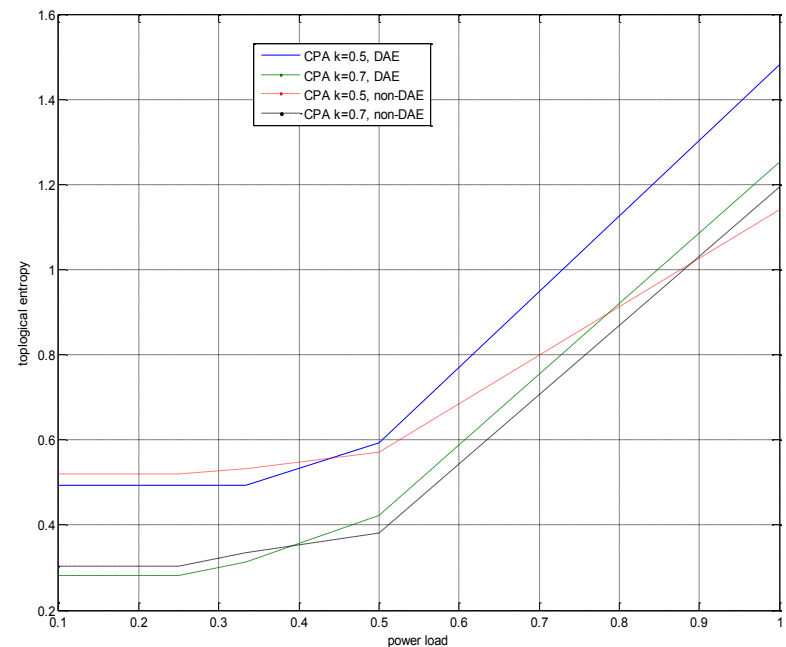

Figure 5: The CPA secure capacity under linear models

For DAE model, we assume the error $e \sim N(0,1)$, then we can adopt the implementation for the model as in [8]: 


$$
\begin{aligned}
& \theta_{p} t= \\
& { }_{j=1}^{19} u_{p j} z_{j_{0}} e^{\lambda_{28+i} t}+{ }_{j=1}^{19} u_{p j}\left[z_{j_{0}} \quad{ }_{k=1}^{19} \quad{ }_{l=k}^{19} h_{0} z_{j_{0}} z_{k_{0}} e^{\left(\lambda_{k}+\lambda_{l}\right) t}\right. \\
& V_{q} t= \\
& { }_{j=1}^{19} u_{q j} z_{j_{0}} e^{\lambda_{n+i} t}+{ }_{j=1}^{19} u_{q j}\left[Z_{j_{0}} \quad{ }_{k=1}^{19}{ }_{l=k}^{19} h_{0} Z_{j_{0}} Z_{k_{0}} e^{\left(\lambda_{k}+\lambda_{l}\right) t}\right.
\end{aligned}
$$

where $\theta_{p} t, V_{q} t$ are the voltage magnitude and phase at each bus. $\lambda_{i} \mathrm{~s}$ are the eigenvalues of the $A(t)$ as in equation (23) and $h_{0}$ is the power factor. The detailed definition of other parameters can be checked in [8]. For the convenience of the simulation, we set $z_{j_{0}}=z_{k_{0}}=1, u_{p j}=u_{q j}=0.5$. The simulation results are presented in Figure 6 . This time, we can see that the topological entropy has significant difference comparing with the Figure 4 and Figure 5. However, the tendencies of the curves are similar. Also, from the Figure 6, we can still conclude that CPA secure capacity is smaller than eavesdropping secure capacity.

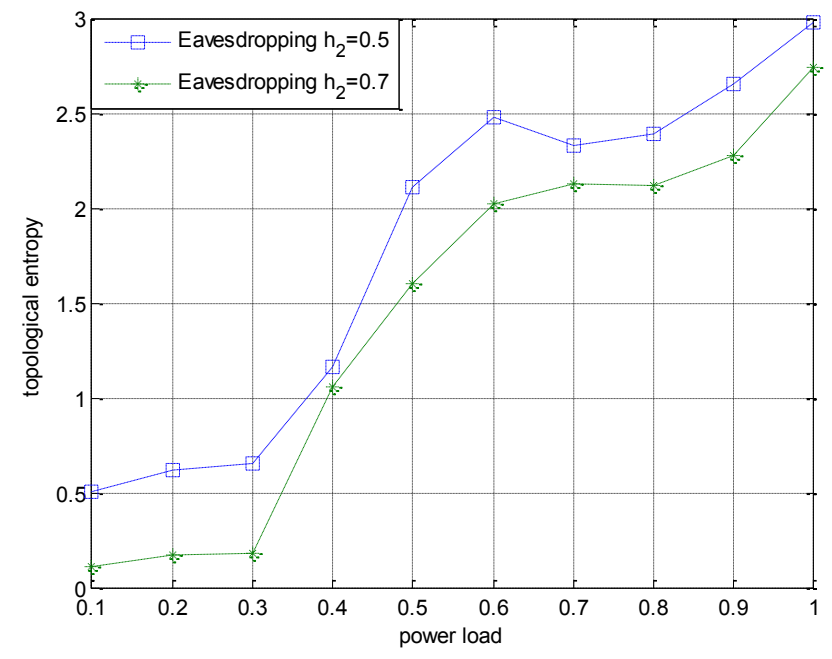

Figure 6: The eavesdropping secure capacity under DAE model

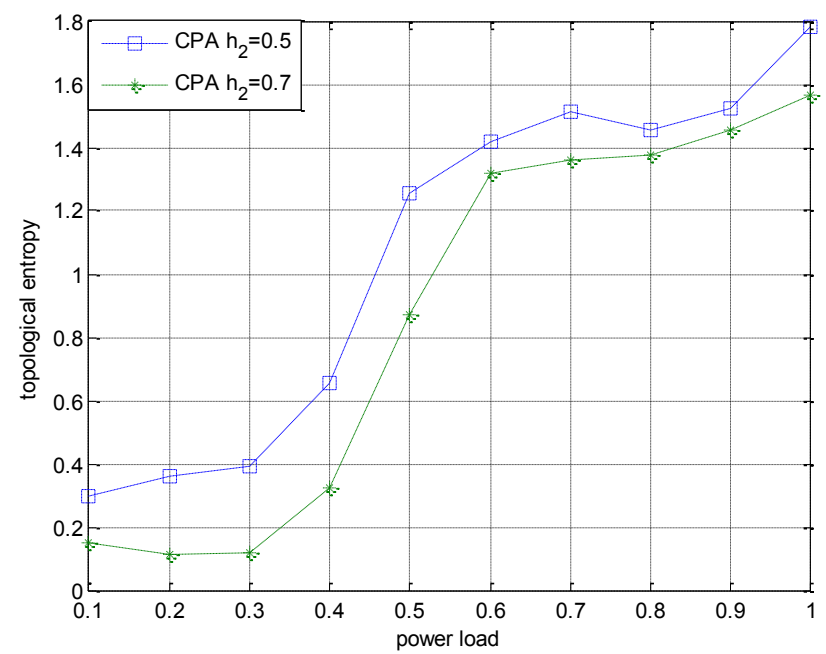

Figure 7: The CPA secure capacity under DAE model

\section{CONCLUSION}

In this paper, we find out the upper bound and lower bound for secure and reliable communication capacity under
CPA attack. By considering DAE power system model, we derive an analytical formula to calculate the topological entropy. Simulation results show that even though there is a significant difference between the results under linear model and the DAE model, the overall tendencies are matched. The results of this research can provide an important theoretical basis for the future implementation of smart grid system, especially for protocol design within WAN.

\section{ACKNOWLEDGMENT}

This work was supported in part by the National Science Foundation of USA (\#1032567), and in part by the National Science Foundation of China (\#61001146).

\section{REFERENCES}

[1] X. Wang, P. Yi, "Security Framework for Wireless Communications in Smart Distribution Grid," IEEE Trans. on Smart Grid, Vol. 2, pp. 809-818, 2011;

[2] Y. Gobena, A. Durai, M. Birkner, V. Pothamsetty, V. Varakantam, "Practical architecture considerations for Smart Grid WAN network," 2011 IEEE/PES Power Systems Conference and Exposition (PSCE), pp. 1- 6, 2011;

[3]Y. Liang, H. V. Poor and S. Shamai, Information Theoretic Security, Now Publishers Inc., 2009;

[4] A. Khisti and G. Wornell, "Secure Transmission with Multiple Antennas I: The MISOME Wiretap Channel," IEEE Trans. on Information Theory, vol. 56, no. 7, 2010;

[5] Y. Liang, H. V. Poor, and S. Shamai, "Secure communication over fading channels," IEEE Trans. on Inf. Theory, vol. 54, pp. 2470 2492, Jun. 2008;

[6] H. Li, L. Lai, "Communication Capacity Requirement for Reliable and Secure State Estimation in Smart Grid," $1^{\text {st }}$ SmartGridComm, IEEE, 2010;

[7] F. L. Alvarado, The Dynamics of Power System Markets, Department of Electrical Computer Engineering, University of Wisconsin, Madison, WI, Tech. Rep. PSERC-91-01, Mar, 1997;

[8] I. Martinez, "A Structure-Preserving Approach to Power System Normal Form Analysis," Power Tech, IEEE, Lausanne, 2007.;

[9] I. Hiskens, "Significance of Load Modeling in Power System Dynamics," X Symposium of Specialists in Electric Operational and Expansion Planning, Brazil, May, 2009

[10] K. Kurosawa, T. Takagi, "One-Wayness Equivalent to General Factoring," IEEE Trans. on Information Theory, vol.55, pp. 42494262, 2009;

[11] B. Bahrak, M.R.Aref, "Impossible differential attack on sevenround AES-128," IET Information Security, vol.2, pp. 28-32, 2008;

[12] X. Song; L. Wang, "Alder's topological entropy of generalized discrete dynamical system," 2012 International Conference on Systems and Informatics (ICSAI), pp. 63-65, 2012;

[13] B. Schneier, Cryptography. Secrets \& Lies: Digital Security in a Networked World, Wiley Computer Publishing Inc., 2000;

[14] A. S. Matveev, A. V. Savkin, Estimation and Control Over Communication Networks, Birkh auser, 2009;

[15] J. Nutaro and V. Protopopescu, "The impact of market clearing timeand price signal delay on the stability of electric power markets," IEEE Trans. on Power System, vol. 24, pp. 1337-1345, 2009.

[16] A. Abur, Power System State Estimation: Theory and Implementation, Macel Dekker. Inc., 2004. 